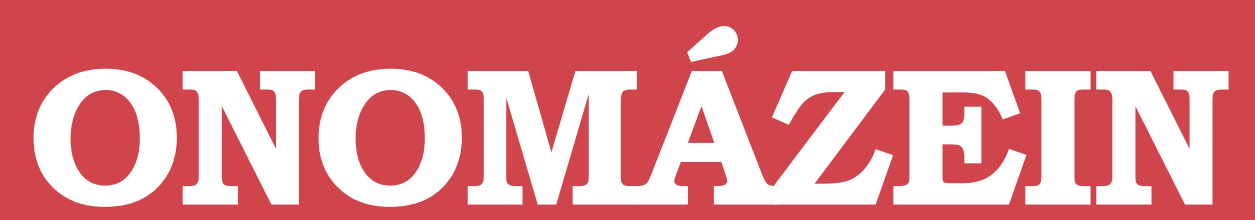

Revista de lingüística, filología y traducción
PONTIFICIA UNIVERSIDAD CATÓLICA DE CHILE FACULTAD DE LETRAS

\title{
Gramática para hablantes de la lengua purépecha: variación dialectal y estándar ${ }^{1}$
}

Grammar for P'urhepecha speakers:

dialectal variation and standard

\section{Cristina Monzón \\ El Colegio de Michoacán \\ México}

Número especial

Las lenguas amerindias en Iberoamérica: retos para el siglo XXI

2017
ONOMÁZEIN - Número especial

Las lenguas amerindias en Iberoamérica: retos para el siglo XXI (2017): 97-114 DOI: 10.7764/onomazein.amerindias.06

\section{(c) $($ i $)$}

Cristina Monzón: Centro de Estudios Antropológicos, El Colegio de Michoacán, México.

| Correo electrónico: cmonzon@colmich.edu.mx

Fecha de recepción: octubre de 2016

Fecha de aceptación: abril de 2017 


\section{Resumen}

La lengua purépecha se habla en el estado de Michoacán, en México. Se trata de una lengua aglutinante que muchos hispanohablantes tildan despectivamente de "dialecto". Desde el punto de vista lingüístico, se han identificado diferentes variedades del purépecha que mantienen, no obstante, un elevado grado de comprensibilidad. Con el doble propósito de darle prestigio a la lengua y de dar testimonio de su riqueza, se está elaborando una gramática dirigida a hablantes del purépecha. Este artículo presenta un ejemplo del método utilizado para facilitar la adquisición escrita de la lengua a los hablantes de las diferentes variedades.

Se presenta una lección de las que componen la gramática en elaboración a fin de que toda la comunidad lingüística purépecha tenga acceso al estándar, de reciente implementación. La variación de cada morfema se articula a través de ejercicios que llevan al lector purépecha a identificar la forma de dicho morfema en el habla de su comunidad asociándola con la variante estándar. Por otra parte, debido a que la gramática de la lengua nunca se ha enseñado en la escuela, de forma explícita, se introduce paulatinamente al lector en conceptos básicos de teoría gramatical. En esta gramática, el purépecha es la lengua empleada y ejemplificada con oraciones y extractos de textos, mientras que el español se utiliza para las explicaciones y definiciones. Se emplea un método de corte comunicativo para la enseñanzaaprendizaje de la lengua.

Palabras clave: purépecha; México; morfología; gramática; variedad estándar.

\section{Abstract}

The P'urhepecha language is spoken in the state of Michoacan, Mexico. It is an agglutinative language referred to by many Spanish speakers discriminatorily as "dialect". From a linguistic point of view, different varieties of the language have been identified, though there is an acceptable level of comprehension between speakers from the diverse varieties. With the double aim of giving prestige to the P'urhepecha language and illustrating its richness, a grammar is being developed for native speakers. This article presents a lesson of the grammar in process so as to illustrate the methodology used to facilitate the acquisition of the written

1 Agradezco a Carla Amorós las correcciones y sugerencias que permitieron mejorar este artículo. 
code, the standard, recently implemented. The variation of each morpheme is supported by several exercises that enable the P'urhepecha reader to successfully identify the morpheme in their own variety and at the same time to associate it with the standard variant. Furthermore, given the fact that the grammar of the language has never been taught at school, basic grammatical concepts are gradually introduced. In this grammar, P'urhepecha is the language employed for examples, sentences and texts, while Spanish is used to explain the grammar and give definitions. A communicative approach is selected to the learning and teaching of P’urhepecha.

Keywords: P’urhepecha; Mexico; morphology; grammar; standard variety. 


\section{Introducción}

En el estado de Michoacán, México, se ubica una comunidad purépecha que se concentra en cuatro regiones conocidas como la zona lacustre: la sierra, la cañada de los once pueblos y la Ciénega. Se calcula que esta población cuenta con alrededor de 130000 hablantes, cuya variación dialectal diferencia unas comunidades de otras. Curiosamente, los pocos estudios dialectales con los que se cuenta a fecha de hoy no delimitan claras isoglosas (Friedrich, 1971; Chávez Rovadeneyra, 2004).

Aun cuando la diversidad dialectal es muy amplia, el purépecha se considera lingüísticamente una única lengua, dado que la comunicación entre los hablantes de las distintas comunidades es relativamente fluida (consúltese el Catálogo de las lenguas indígenas nacionales, 2008). Sin embargo, es notoria la predilección por el uso del castellano para agilizar el intercambio lingüístico, gracias al alto grado de bilingüismo de los purepechahablantes. Parece observarse que los cambios de pronunciación y de léxico, fundamentalmente, que caracterizan a las distintas comunidades de habla llevan a los hablantes a inquirir constantemente sobre el significado de alguna palabra o expresión en concreto. A lo anterior se suma la ausencia de vocabulario para expresar en purépecha muchos aspectos de la vida moderna y, en particular, aquellos relacionados con el gobierno, la ley, las organizaciones sindicales, etc.

La práctica lingüística de la lengua purépecha es predominantemente oral, con una disponibilidad de material de lectura muy limitado. Varias instituciones educativas (Secretaría de Educación Pública [SEP], Instituto Nacional de Lenguas Indígenas [INALI], la Universidad de San Nicolás de Hidalgo, por ejemplo) han implementado para sus publicaciones sus propios alfabetos, de forma que hasta el momento se cuenta con alrededor de 5 alfabetos, que presentan, no obstante, diferencias mínimas - la opción $\langle n\rangle,\langle n h\rangle 0\langle\eta\rangle$; la representación de las consonantes intervocálicas oclusivas aspiradas como $\left\langle p^{\prime}\right\rangle,\langle$ 'p $\rangle,\langle j p\rangle$, que en posición inicial solo tiene la representación 〈p'> etc. - . Así pues, la elección de las distintas grafías depende de la institución educativa en la cual se aprendió a escribir así como del momento en que se llevó a cabo el aprendizaje.

Esta diversidad de alfabetos es el resultado de más de 50 años de acaloradas discusiones sobre la mejor forma de representar ortográficamente la lengua. La discusión en torno al principio fonémico, esto es, la deseable correspondencia biunívoca entre grafemas y fonemas, continúa presente hoy día bajo el término de "normalización de la lengua” y con la iniciativa del INALI. A la mencionada diversidad de alfabetos se une la creatividad del propio individuo, quien, en un afán por representar su propia variante, desarrolla símbolos o combinaciones de letras percibidas como más representativas del sonido característico empleado en su particular comunidad de habla.

En este contexto, y con el fin de elaborar una gramática del purépecha, se argumenta la necesidad de articular una obra que se ocupe simultáneamente de la variación dialectal de la 
lengua y la propuesta del estándar, de reciente implementación (Monzón y Roth, 2011). Este proyecto aspira a proveer a los hablantes de la lengua originaria de un medio para reflexionar sobre su propio idioma y para familiarizarse con una representación ortográfica unánime. A través de la práctica, se busca mostrar la diferencia entre la utilización del alfabeto para transcribir el habla oral y para representar una variedad "común", comprensible para la comunidad lingüística amplia, que sobrepasa los límites de cada pueblo. Se considera, además, que la codificación gramatical podría ser un instrumento viable para frenar la discriminación basada en el prejuicio de que el purépecha "es un dialecto, no una lengua".

\section{La gramática y su contenido}

La lengua purépecha es una lengua aglutinante que cuenta con más de 60 morfos distintos para la formación de la palabra. Es, pues, el estudio centrado en la morfología el ámbito fundamental al que se dedicará esta gramática. Su propósito es reunir el conocimiento alcanzado por investigadores de la lengua desde el siglo XVI hasta la fecha² en una forma y formato accesible para su comprensión por hablantes con una educación básica. Por lo tanto, resulta de vital importancia utilizar un metalenguaje próximo a la vida cotidiana sin, por ello, simplificar los aspectos gramaticales que se enseñan.

A este respecto, es importante resaltar que no se contempla apoyar la explicación del conocimiento sobre la lengua castellana adquirido en la formación primaria, debido a que este conocimiento no es confiable y transferible, puesto que está asociado con estructuras totalmente distintas a la de la lengua originaria. La formación en competencia gramatical deberá lograrse a través del control de los temas que se expondrán de tal manera que el aprendizaje esté secuencialmente graduado.

Los morfemas que se estudiarán se distribuirán en tres libros. El primero agrupará toda aquella morfología que aporta información semántica pero que no afecta al plano sintáctico. Comprenderá alrededor de 30 morfemas y constará de una introducción básica de terminología gramatical que agilizará la exposición. El segundo libro se enfocará en los, aproximadamente, 40 morfemas de espacio que informan tanto sobre las partes del cuerpo humano como de partes de otros cuerpos que conforman el mundo que nos rodea. Es aquí donde se iniciará la formación del lector en el análisis sintáctico de una oración sencilla en purépecha.

2 Las artes de Gilberti (2004 [1558]) y Lagunas (1983 [1574]) del siglo XVI; para el XVIII la de Basalenque (1994 [1714]); en el siglo XIX la gramática de Grasserie y León (1896) y la de Nájera (1944 [1879]). Para el siglo XX Foster (1969) y para el XXI Chamoreau (2000). Con estas obras la presentación de la gramática en su conjunto concluye, la nueva tendencia investigativa se enfoca hacia el análisis de temas específicos siendo notorio el incremento de estudiosos de la lengua en este siglo. 
La exposición gramatical se verá limitada a la información que requieren los morfemas espaciales en su interacción con la estructura de la oración. Finalmente, el tercer libro se dedicará a aquellos morfemas que no solo aportan mayor significado, sino cuya presencia puede modificar la sintaxis de la oración, cuando la palabra objeto de estudio es un verbo. Este grupo de morfemas es pequeño, alrededor de 8, aunque su complejidad es bastante elevada. Todos ellos se vinculan estrechamente con la sintaxis, si bien solo unos pocos presentan una sorprendente capacidad de cambio de la estructura sintáctica cuando forman parte de un verbo.

Según se avanzó anteriormente, la gramática está redactada en castellano, la metalengua, si bien la ejemplificación estará en purépecha. El problema que se planteó es la adopción de una determinada postura respecto a la representación gráfica de la lengua originaria: por un lado, había que seleccionar un alfabeto determinado de entre los cinco que existen y, por otro, escoger el dialecto purépecha que serviría como base o establecer una alternativa. A este respecto, se decidió que todo aquello que se escribe en purépecha en esta obra se hiciera utilizando la convención del p’urhepechamárko (Monzón, 2014), estándar propuesto en varias publicaciones y presentaciones en el 2011 y puesto en práctica en el libro de cuentos tradicionales Tsípiakuarhip’urhepechauantanskuajimpo (Monzón y Diego, 2013). Obviamente, dado que dicho estándar no goza actualmente de un uso generalizado, la exposición se articulará, como veremos, con una práctica didáctica para que el estudiante identifique los morfemas en uso en su propia variedad vernácula.

\section{Metodología gramatical}

Acceder con un único manual de gramática a lectores cuya práctica oral está limitada a la variedad utilizada en sus respectivas comunidades plantea un verdadero reto. La lección que se ilustrará más adelante muestra la propuesta seguida, que se sustenta en dos elementos principales: ejercicios de reescritura iniciales y gramática inductiva para activar el proceso de descubrimiento.

Los ejercicios tienen dos fines. Por un lado, al principio de la lección, llevar al estudiante a identificar su propia variante del morfema estudio a través de la reescritura de una selección de ejemplos. Con esta práctica se busca asegurar la total comprensión de los ejemplos sobre los cuales se trabaja. Por otra parte, se espera que el lector logre establecer a través de la práctica de los ejercicios la asociación entre la representación escrita estándar y la forma dialectal de la práctica oral y escrita del lector, de tal forma que se familiarice con la variante erigida como estándar.

Los ejercicios inducen así al lector a iniciar una reflexión sobre el significado que el morfema en cuestión está aportando. Se busca que el estudiante intuitivamente formule hipótesis sobre la función que tienen los distintos sufijos. Para ello, se servirá del contraste entre 
oraciones - con presencia/ausencia del sufijo - que serán discutidas, lo que le permitirá establecer el significado gramatical tras realizar el pertinente análisis sintáctico. Terminado el proceso de descubrimiento, a través de ejercicios y discusión de pares de oraciones contrastivas se ofrecerá la regla gramatical, así como el nombre con el cual se identifica el morfema. Básicamente el mismo proceso de explicación se retoma para ilustrar las excepciones a la regla u otras características gramaticales del sufijo estudiado. Los términos e información esencial para el estudio de la morfología así como los procesos de análisis sintáctico se exponen en páginas resaltadas en amarillo que aparecerán dispersas por el manual, antes de que el conocimiento que se imparte lo requiera para el estudio de un morfema en particular.

En resumen, la exposición de la gramática con enfoque en la morfología se divide en tres partes que van desde los morfemas más sencillos — aportación semántica- a los más complejos -aportación semántica y sintáctica-.

\section{TABLA 1}

Esquema de la explicación gramatical de cada lección

\begin{tabular}{|ll|}
\hline - & Identificación del morfema en la propia variante \\
0 & Hipótesis del estudiante \\
- & Explicación gramatical \\
0 & Introducción del concepto gramatical \\
0 & Regla gramatical \\
- & Nombre asignado al morfema \\
• & Excepciones o ampliación de las características gramaticales \\
$=$ identificación del morfema en nuevo contexto \\
- & Explicación gramatical \\
& Advertencia sobre morfemas homófonos \\
\hline
\end{tabular}

\section{El contenido de una lección, ilustración}

Se presentan, a continuación, las bases para la producción de esta gramática "práctica" mediante el ejemplo de una lección que se ha elaborado y para la cual los conocimientos que se presuponen han sido adquiridos a través del primer y del segundo libro.

\subsection{Conocimientos presentados en lecciones anteriores}

En el libro I, que presenta clíticos y sufijos que aportan información semántica independiente de la estructura sintáctica, se han introducido explicaciones sobre términos gramaticales. Antes de leer la presente lección, el estudiante conoce el significado de "morfema”, "sufijo", 
"clítico", "sustantivo", "frase”, "verbo" y "oración”, términos que se ilustran de forma que el hablante adquiera conciencia de la característica aglutinante de la morfología del purépecha. Así, no es infrecuente que la utilicen para impresionar al curioso que hace preguntas sobre el purépecha o para retar al lingüista que lo enseña. Igualmente en este libro se presentan los pronombres personales nominativo y nominativo-acusativos tanto en plural como en singular. Para estos pronombres fue necesario introducir el análisis sintáctico de una oración con verbo transitivo, análisis sintáctico cuya complejidad se incrementa en el libro II, a fin de poder presentar las particularidades sintácticas de los morfemas espaciales.

Para facilitar una consulta rápida, al final del libro se proveerá un vocabulario de términos gramaticales.

Los ejemplos se toman de las tareas que, desde el 2001, he venido recolectando de los estudiantes que han asistido a diplomaturas, cursillos o cursos reglados en centros educativos 3 .

\subsection{La lección sobre el sufijo de concordancia con la frase acusativa en terce- ra persona del plural}

Cada lección del manual se inicia con un encabezado que muestra la posición que ocupa el morfema que se estudiará, marcando la casilla en color:

\begin{tabular}{|l|l|l|l|l|l|l|l|l|l|l|}
\hline Raíz & -1 & -2 & -3 & -4 & -5 & -6 & -7 & -8 & -Flexión & =clíticos \\
\hline
\end{tabular}

Varias líneas más abajo, se inicia la lección con el título. Este ofrece el nombre que se le asigna al sufijo. El color negro se utiliza para la referencia en castellano (acusativo de $3^{\circ}$ persona del plural) e inmediatamente abajo, en verde, la propuesta de nombre en purépecha (3ºúnikuaakusativu).

Seguidamente, en un recuadro central, se indica la forma del sufijo objeto de estudio. Esta forma es la representación gráfica del sufijo en la variante estándar que la obra utiliza. En los recuadros de la derecha se presentan las formas alternativas de dicho morfema que están en uso en diferentes variedades de la lengua (véase la tabla 2).

3 Quiero expresar a todos ellos mi agradecimiento, no solamente por los ejemplos de oraciones utilizadas, sino, sobre todo, por el interés que mostraron en la gramática. En particular, querría destacar a Manuel Sosa, oriundo de la comunidad de Angahuan, quien, como miembro de El Colegio de Michoacán, ha ilustrado incansablemente el uso de su lengua durante años. Gracias a la lectura que ha hecho del Arte de fray Maturino Gilberti, me ha proporcionado un rico acervo de ejemplos y mostrado que mucho de lo dicho en el siglo XVI todavía es conocido en su comunidad, caracterizada por un predominio de monolingüismo entre mujeres y niños. 


\section{TABLA 2}

Representación gráfica de las diferentes variantes del sufijo

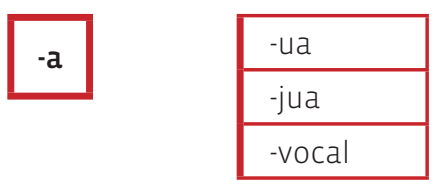

Dada la diversidad dialectal de la manifestación de este sufijo, como segundo encabezado en letras de tamaño más grande se presenta una oración en la cual se resalta tipográficamente el sufijo. La selección de esta oración toma en consideración la factibilidad de su comprensión en cualquier variedad. Inmediatamente después, se ofrecen oraciones que se espera que sean de fácil comprensión para todos y, por lo tanto, que permitan al estudiante familiarizarse con el uso del sufijo (véase la tabla 3).

\section{TABLA 3}

Ejemplificación del sufijo objeto de estudio

Jorhentpirijorhenkuarhirichanijorhentaaxati

Ejemplos con el sufijo-a:

José charakuchanixench’aasïnti (José ama a los bebés)

T’arhépititakichanikauaruuaxanhaasti (El anciano sentó a los niños adentro de la barranca)

Ta kúraimariakolituchaniiámukuinaraasti (El sacerdote mandó cortarles el pelo a todos sus acólitos)

Nótese que en todos los ejemplos seleccionados la vocal que precede al sufijo bajo estudio es $\langle a\rangle^{4}$. La razón para limitar el contexto a la vocal $\langle a\rangle$ es reducir las alternativas dialectales a tres opciones: la forma -a del estándar, que es, a la vez, la utilizada en un amplio número de variantes, y dos alternativas más, la de aquellos dialectos que usan -ua y la de aquellos que usan -jua. En esta gramática, con el fin de que los hablantes identifiquen la forma del sufijo en su vernáculo, se solicita que reescriban las oraciones que se han proporcionado (véase la tabla 4).

Se presupone aquí que el estudiante, al reescribir algunos ejemplos, inicia su propia reflexión y busca implícitamente establecer una hipótesis sobre el significado de dicho

4 La identificación del sufijo en negritas y la traducción no aparecerá en el original, se anota aquí para beneficio de los lectores de este artículo. 


\section{TABLA 4}

Ejemplo de ejercicio

EJERCICIO 1. >> Reescribe tres de los ejemplos dados en tu propia variedad: el sufijo -a podría presentarse como -ua o -jua.

¿Ya reconociste el sufijo en el habla de tu comunidad?, escribe cuál es:

sufijo. Reiteramos que en esta gramática las oraciones ejemplo en purépecha no van acompañadas de su traducción al castellano, ya que dicha traducción no aporta a la comprensión y sí ofrece el riesgo de confundir al bilingüe, al requerirle que trabaje simultáneamente con dos lenguas muy disímiles.

El ejercicio descrito ha guiado al estudiante 1) en el proceso del descubrimiento e identificación del sufijo y 2) en la percepción de su significado. A continuación, la gramática proporciona la respuesta en un recuadro marcado con una explicación basada en el contraste entre dos oraciones - con y sin presencia del sufijo objeto de estudio- Se hace hincapié en la diferencia de significado y, para este caso, en la relación entre frase sustantiva plural y la presencia del sufijo5. Es aquí cuando se introduce el concepto de concordancia entre verbo y frase.

Seguidamente, se presenta el análisis sintáctico de la oración ejemplo que ha encabezado esta lección, dada su pertinencia para la comprensión del sufijo de concordancia-a (véase la tabla 5).

Téngase en cuenta que a esta exposición le preceden dos lecciones sobre la función sintáctica de los casos nominativo, acusativo, locativo, etc., tratadas en los libros I y II. Es importante mencionar que la selección de los nombres de la función sintáctica está motivada por dos factores: por un lado, el purépecha es una lengua de casos (nominativo, acusativo, genitivo, locativo, residencial y, en algunas variantes, comitativo e instrumental); por otro lado, se halla una razón meramente utilitaria y pragmática, esto es, evitar los términos "sujeto", “objeto”, etc., que permite eludir la formación, generalmente deficiente, de la enseñanza de la gramática castellana e inhibe la búsqueda por parte del estudiante de establecer un paralelo entre el español y el purépecha.

5 Este análisis se encuentra ya en las obras de los siguientes autores: Basalenque (1994 [1714]: 139), Grasserie y León (1896: 61), Friedrich (1984: 76) y Monzón (1997: 40). 


\section{TABLA 5}

Ejemplo de análisis sintáctico

Jorhentpirijorhenkuarhirichanijorhentaaxati

(El maestro les enseña a los alumnos)

\section{Análisis sintáctico}

\begin{tabular}{|l|l|l|l|}
\hline 1 $^{\text {er }}$ paso & identificación del verbo & jorhentaaxati & verbo \\
\hline $2^{\text {o }}$ paso & ¿Ne jorhentaaxakijorhenkuarhirichani? & Jorhentpiri & frase nominativa \\
\hline $3^{\text {er }}$ paso & ¿Néchanijorhentaaxakijorhentpiri? & jorhenkuarhirichani & frase acusativa \\
\hline
\end{tabular}

La presencia del sufijo -a es obligatoria cuando en la frase con función acusativo se habla de varias personas, santos, dioses antiguos o sea de seres racionales

Llamaremos a este sufijo -a 'acusativo plural'

En este contexto, la presentación del análisis sintáctico permite mostrar que la concordancia de número se lleva a cabo entre la frase con función acusativa y el verbo. Seguidamente, en un recuadro rojo se resalta la regla gramatical y se le asigna un nombre al morfema estudiado.

Dado que en algunas variantes el sufijo -a armoniza con la vocal que le precede, puede convertirse en:

-i cuando le precede la vocal \{i\} como en Ji p’orhempiiskapámp’irichani.

-e cuando le precede la vocal \{e\} como en Imaexeestipámp’irichani.

-o cuando le precede la vocal \{0, u\} como en Imapirekooskauáchachani.

La lección ofrece nuevos ejemplos y solicita al estudiante que los reescriba con el fin de identificar la particularidad de su variante. Habiéndose logrado esta nueva identificación de la forma del sufijo -a, se le solicita al estudiante que aplique la regla gramatical sobre las oraciones del ejercicio. Para beneficio del lector, igual que hemos resaltado con negritas el sufijo -a, se ha subrayado la frase acusativa (véase la tabla 6).

\section{TABLA 6}

Ejemplos con el sufijo -a en otros contextos

Uarhititumpichaniexeasti (La señora está mirando a los muchachos).

Nana k’érip'amok'uasïntinimakuajempachani (La abuelita le tiene lástima a sus nietos).

Ts'iuérati sapichujirikuarhikuastiimaritatachani (El niñito se les escondió a sus papás). 
Cuando hay excepciones a la regla general, los temas se introducirán con preguntas del tipo “¿Qué sucede cuando en la frase acusativa se enuncia animales o cosas?”. Los ejercicios planteados, así como la inclusión de un conjunto de oraciones y un extracto de un texto, buscan ofrecer la información necesaria para que el estudiante mismo realice el proceso de descubrimiento. Se culmina con la exposición de la regla de excepción en otro recuadro (tabla 7).

\section{TABLA 7}

Ejemplo de presentación de la regla gramatical con excepción

\section{Al hablar sobre animales y cosas}

El purépecha no obliga necesariamente a que las palabras se pongan en plural, por lo que el verbo generalmente tampoco presenta el sufijo de acusativo plural.

Dadas las características del purépecha, varias consideraciones se presentan para mostrar las particularidades de la concordancia cuando se habla de animados racionales e inanimados. Así, la concordancia de número no se da cuando la frase acusativa se refiere a la $1^{\mathrm{a}}$ y $2^{\mathrm{a}}$ personas del plural:

Tata jorhentpirichaíntskustiksïniíntsïnperatamach’anksïni (Los maestros les dieron un premio a ustedes).

Juchitinántit’irerastits'ïnitatsïnikakuiripita (Mi mamá nos dio de comer frijoles con carne).

Lo anterior permite redefinir la regla y dar el nombre definitivo que tendrá el morfema-a (tabla 8).

\section{TABLA 8}

Cuadro sinóptico de la regla gramatical

Como se puede observar en todas las oraciones, la frase acusativa receptora se refiere a los pronombres personales de $1^{\text {a }}$ persona del plural juchants'îni o al clítico pronominal =ts'ïni así como a la $2^{\mathrm{a}}$ persona del plural ch’anksïnio al clítico pronominal =ksïni. Aun cuando ambos se refieren a entes animados en plural, en el verbo no se lleva a cabo la concordancia de número.

El sufijo -a recibirá entonces el nombre de 'acusativo de $3^{\text {a }}$ plural'

6 La especificación de que el sufijo de acusativo plural solo concuerda con la tercera persona de plural se menciona en los estudios de los siguientes autores: Wolf (1989: 139), Chamoreau (2000: 72), Villavicencio Zarza (2006: 119), Capistrán Garza Bert (2010: 64) y otros más. 
La particularidad de la regla merita insistir para asegurarse de que el estudiante la comprendió, por lo que se ofrece un paradigma que permite visualizar nuevamente la diferencia. En contraste, cuando se trata de la tercera persona del plural, la concordancia verbal sí se lleva a cabo, tal y como se puede apreciar en la siguiente oración:

Tatakasapichunanakachanijupik’uasti (el niño agarró de la mano a las niñas).

Nótese el contraste con:

Tatakasapichujupik’ustitsïni (juchats'ïni) (el niño nos agarró).

Tatakasapichujupik’ustiksïni (ch’anksïni) (el niño los agarró a ustedes).

Tatakasapichujupik’ustirini (jinteni) (el niño me agarró).

A través de un ejercicio se pide que el estudiante constate la regla gramatical en varios textos (tabla 9).

\section{TABLA 9}

Ejemplo de ejercicio para consolidación de la regla gramatical

EJERCICIO 10. \> Subraye los clíticos pronominales nominativo acusativo =ts'ini y =ksïni en los siguientes textos e identifique el verbo de la oración. Note la ausencia del morfema de acusativo plural -a.

Finalmente, debido al amplio número de sufijos homófonos es pertinente que el hablante sea consciente de ello y aprenda a diferenciarlos, tanto por el significado que percibe como por la posición en la cual se ubican en la cadena sufijante de la palabra. Por ello, se ofrece una advertencia sobre los morfemas -a, los cuales fueron presentados en los dos volúmenes precedentes. Se trata del sufijo -a (-ua en otras variantes) conocido en la literatura como futuro y que Friedrich correctamente identifica cuando describe su referente: "Refiere a intenciones, potencialidades, posibilidades, predicciones y creencias sobre el futuro"; y del sufijo-a que, al igual que en el caso anterior, alterna con -ua, pero que difiere dado que dicha alternancia se da dentro de una misma variedad. Este sufijo forma parte del conjunto de más de 40 morfemas que el purépecha posee para indicar un espacio determinado que se ubica en un cuerpo, ya sea este de persona o animal o de entidades creadas por el hombre o por la naturaleza (Monzón, 2004; Nava López, 2004; Capistrán Garza Bert, 2010) (véase la tabla 10).

7 "Refers to intentions, potentials, possibilities, predictions, and beliefs about the future" (1984: 70). 


\section{TABLA 10}

Ejemplo de ejercicio para consolidación de la regla gramatical

\section{Cuidado:}

No confunda el sufijo -a de acusativo plural con los sufijos -a de futuro y -a de morfema de espacio. Hay diferencias entre ellos: 1) por su posición en la cadena derivacional y 2) por su significado:

Ejemplo de -a de futuro:

Amampauátsïniuanenkuasïrikukunt’aati (La mamá le arreglará el guanengo a su hija).

Ejemplo de -a de morfema de espacio

Tsinap'iritumpinip’áakuxati (El doctor le está tocando la panza al niño).

Se concluye con un resumen de las reglas y un último ejercicio que pide al hablante su propia reflexión sobre su variedad nativa. Esta lección en particular añade información del siglo XVI proveniente de las Artes de la lengua de Michoacán, elaboradas por los franciscanos fray Maturino Gilberti y fray Juan Baptista de Lagunas. Es una referencia histórica que generalmente no aparece en las lecciones, pero que consideramos pertinente para aquellos casos en los que el estado de la lengua en la antigüedad es relevante para que el estudiante comprenda la variabilidad que existe para el uso del plural en sustantivos y la concordancia de número con frase acusativa. La historia de la lengua purépecha permite entender por qué hoy en día todavía es posible no utilizar el plural con referentes que son cosas, mientras que con animales sí se utiliza en ocasiones (véase la tabla 11).

\section{El p’urhepechamárko como medio para la comprensión lectora}

En la actualidad, se observan dos prácticas, que se realizan frecuentemente por los hablantes de la lengua que buscan lograr una comunicación escrita fluida. Por un lado, se percibe que aquellos que en la producción oral han sustituido la trillada retrofleja [rh] por una lateral [I], en sus escritos optan por la representación de 〈rh〉 en la mayoría de los casos. Por otro lado, ha Ilamado nuestra atención la práctica de autores-cantantes que, debido a la dificultad de comprensión que tiene su variedad en el resto de la región, modifican el texto de sus canciones para lograr que la letra sea accesible a la mayoría de los receptores. A lo anterior pueden añadirse los testimonios que algunos estudiantes, tanto de la Universidad Intercultural Indígena como de la Escuela Normal Superior, han hecho respecto a la necesidad que tuvieron de modificar su forma de expresión con la finalidad de que sus compañeros pudiesen entenderlos.

Si bien un estudio de las diversas adaptaciones realizadas individualmente en un contexto escolar o el desarrollo de la escritura en la comunicación por teléfonos celulares arrojaría luz sobre la práctica de la escritura, la factibilidad de realizar una investigación de tal enver- 


\section{TABLA 11}

Ejemplo de apartado de referencia histórica

\section{HISTORIA}

Fray Maturino Gilbertien su Arte de la lengua de Michoacán (2004 [1558]) así como Fray Juan Baptista de Lagunas en su Arte (1983 [1574]) ejemplifican la presencia del sufijo de acusativo de plural, que, en aquella época, se escribía -hua, forma que se utiliza en Angahuan -jua, pero que en la mayoría de las comunidades hoy en día es -a o presenta armonía vocálica $\left(V^{*}\right)$. Note el lector que estos frailes gramáticos, al dar ejemplos sobre este el sufijo -hua, muestran que su presencia es obligatoria cuando se está hablando de personas, pero que generalmente se encuentra ausente cuando se habla de animales o frutas:

O si yo oviesse enseñado a estos naturales el sancto evangelio

Nondiatinithuynhurendahuapiringapurepechan S<anto>euangelio. (Gilberti, 84)

Vcuhuavuachanthirequa (Haz de comer a los muchachos). (Gilberti, 155)

Hi vandahtsicuhuahacacuiripuechani Yo predico a los hombres. (Gilberti, 155)

“... Estoy guardando las ovejas... Hi xuratengarieranguhuaxaca ... (Gilberti, 128)

<Puqui echa>Ahpenschaxahuanstitzimancauallo, tanimu, thamu, temben, macataricauallo.los leones comieron dos o tres cavallos, 〈cuatro, diez, veinte caballos> (Gilberti, 128)

Hi cacanarinscavandangucata Yo quebrante los mandamientos. (Gilberti, 270)

Ambaruzcanixangari A limpiar los caminos o calles. (Gilberti, 271)

Hucàmarhitinaranjas, Turaznos, i. [e.] Tiene multitud

de naranjas, duraznos, etc. (Lagunas, 187)

gadura es más reducida por el momento y hay que conformarse con crear una propuesta sustentada en el trabajo de los cantautores de gran renombre en toda la región. El reconocimiento otorgado a la producción artística en la comunidad de habla purépecha asegura el reconocimiento de la capacidad comunicativa del purépecha utilizado en estas pirecuas o canciones purépechas.

El resultado del análisis de las características de la letra de las canciones mostró que los compositores restituyeron consonantes y vocales que su variante había perdido. Por otra parte, algunas vocales fueron sustituidas por aquellas que se encuentran en uso en la mayoría de los dialectos. Este esfuerzo sienta las bases para llevar más allá la propuesta y acercarse a un estándar.

Considerando que la lengua tiene una estructura aglutinante, fue posible centrarse en los sufijos para determinar en cada caso la variación dialectal que presentaban. A partir de 
entonces, se seleccionó la forma del sufijo más usada en la mayoría de las variedades o la forma más simple del morfema, o se tuvieron en cuenta ambos criterios, según ocurrió en el caso del sufijo de acusativo de plural cuya lección se ilustró aquí. La propuesta resultante se implementó en un pequeño libro de relatos orales provenientes de tres comunidades, Angahuan, San Lorenzo y La Cantera. La edición realizada de Tsípikuarhip’urhepechauantansku ajimpo (Monzón y Diego, 2013) unifica la representación gráfica siguiendo los términos de la variedad estándar. El resultado parece haber sido positivo, a juzgar por los comentarios que he recibido de hablantes de muy diversas comunidades que han podido leer y comprender los textos. El desarrollo de este estándar está en sus primeras fases de implementación y se espera que la comunidad lingüística global logre solventar sus diferencias dejando a un lado su orgullo comunal respecto a la propia variedad en aras de una variedad de comunicación común a todos.

\section{Conclusión}

La existencia de un amplio número de variedades de una lengua y en casos extremos de lenguas emparentadas plantea problemas si consideramos la necesidad de producir, por ejemplo, 300 ejemplares diferentes de una misma obra para satisfacer las 300 variedades reportadas.

La tendencia al desuso de las lenguas originarias en beneficio de la lengua castellana obliga a buscar medios creativos que le otorguen su indudable mérito y prestigio que les ha sido negado durante siglos, con el fin de luchar por el mantenimiento de su uso y el apoyo a la población indígena y la creatividad humanas, cuyo testimonio está plasmado en dichas lenguas. Encontrar formas que ofrezcan al hablante la posibilidad de uso en ámbitos amplios, no restringidos a su particular comunidad, resulta un paso necesario.

El proyecto de elaboración de una gramática práctica de la lengua purépecha busca, pues, proporcionar conocimiento lingüístico a los hablantes y espera que, si recibe prestigio por parte de los hablantes, sea un grano de arena para el mantenimiento de esta lengua amerindia.

\section{Bibliografía citada}

Basalenque, Diego, 1994 [1714]: Arte de la lengua Tarasca, Dispuesto con nuevo estilo, y claridad, por el R. P. M. Fr. Diego Basalenque, del Orden de N.P. S. Agustin, Provincial que fue de la Provincia de Michoacan y su chronista. Introducción histórica y preparación fotográfica del texto por J. Benedict Warren. Zamora, Michoacán, México: Fimax Publicistas Editores.

Capistrán Garza Bert, Alejandra, 2010: Expresión de argumentos, funciones gramaticales y transitividad en p’orhépecha. Tesis de doctorado, El Colegio de México, México. 
Catálogo de las lenguas indígenas nacionales, publicado en el Diario Oficial de la Federación el 14 de enero de 2008, México.

Chamoreau, Claudine, 2000: Grammaire du purépecha parlé sur les îles du lac de Patzcuaro (Mexique), Munich: LincomEuropa. Studies in Native American Linguistics, 34.

Chávez Rovadeneyra, David Alberto, 2004: Aproximación a la dialectología de la lengua purépecha. Tesis de maestría, Centro de Investigaciones y Estudios Superiores en Antropología Social, México.

Foster, Mary LeCron, 1969: The Tarascan Language, USA: University of California Publications in Linguistics 56, Berkeley y Los Angeles: University of California Press.

Friedrich, Paul, 1971: "Dialectal Variation in Tarascan Phonology", International Journal of American Linguistics, vol. $37, n^{\circ} 3,164-187$.

Friedrich, Paul, 1984: "From meaning to sound", Handbook of middle American Indians 2, Austin: Texas Press, 56-82.

GILBERTI, Maturino, 2004 [1558]: Arte de la lengua de Michuacan compilada por el muy Reverendo padre fray Maturino Gylberti. Transcripción, edición y notas por Cristina Monzón. Zamora, Michoacán, México: El Colegio de Michoacán y Fideicomiso Teixidor.

Grasserie, Raoul de la, y Nicolás León, 1896: Langue tarasque: grammaire, dictionaire, textes traduits et analysés, Bibliothèque Linguistique Americaine, tomo XIX, París: J. Maisonneuve, Libraire Editeur.

Lagunas, Juan Baptista de, 1983 [1574]: Arte y Dictionario: con otras obras, en lengua Michuacana. Co[m]puesto por el muy R.P Fray Iuan Baptista de Lagunas, Praedicador, Guardian de Sanct Francisco, de la ciudad de Guayangareo, y Diffinidor de la prouincia de Mechuacan, y de Xalisco. Introducción histórica con apéndice documental y preparación fotográfica del texto por J. Benedict Warren. Morelia, Michoacán, México: Fimax Publicistas.

Monzón, Cristina, 1997: Introducción a la lengua y cultura tarascas. De acá para allá: lenguas y culturas amerindias, España: Universitat de València. Departament de Teoría dels Llenguatges.

Monzón, Cristina, 2004: Los morfemas espaciales del p’urhépecha; significado y morfosintaxis, México: El Colegio de Michoacán.

Monzón, Cristina, 2014: “Pirekuechauantasïntiksï 'p’urhepechamárko'. Las enseñanzas de los pireris" en Pedro Márquez Joaquín (coord.): Pirekua, canto poco conocido, México: El Colegio de Michoacán y Consejo para el Arte y la Cultura de la Región P’urhepecha, 111-124.

Monzón, Cristina, y Andrew Roth, 2011: "En busca de un estándar. El caso de la lengua p'urhépecha”, Revista Universos, Revista de Lenguas Indigenas y Universos Culturales 8, 187-202 
Monzón, Cristina, y David Diego, 2013: Tsípikuarhip’urhepechauantanskuajimpo, México: Instituto Nacional de Lenguas Indígenas.

NÁjera, Manuel de San Juan Crisóstomo, 1944 [1879]: Gramática de la lengua tarasca (elaborada en los años posteriores a 1834). Edición fiel de su original autógrafo con una introducción biobibliográfica, notas e índices de Joaquín Fernández de Córdoba, presidente de la sociedad de Geografía e Historia de Michoacán y miembro de varias sociedades científicas nacionales y extranjeras, México: Libros de México, S. en p.

Nava López, Enrique Fernando, 2004: La voz media en p’urhepecha. Un estudio de formas y significados. Tesis de doctorado, Universidad Nacional Autónoma de México, México.

Villavicencio Zarza, Frida, 2006: P’orhepechakasosirátahenkwa: Desarrollo del sistema de casos del purépecha, México: Centro de Investigaciones y Estudios Superiores en Antropología Social y El Colegio de México.

Wolf, Paul de, 1989: Estudios lingüísticos sobre la lengua phorhé, Zamora, Michoacán, México: El Colegio de Michoacán y Gobierno del Estado de Michoacán. 